\title{
Review Of Cost Effectual Content Sharingin Cloud Environment
}

\author{
Aluri Shiva Kumara, Dr Pankaj Kawad Kar ${ }^{\mathrm{b}}$ and Dr. N.Rajasekhar ${ }^{\mathrm{c}}$ \\ a Research Scholar, Dept. of Computer Science \& Engineering, \\ Sri Satya Sai University of Technology \& Medical Sciences, Sehore, Bhopal Indore Road, Madhya Pradesh, India \\ ${ }^{b}$ Research Guide, Dept. of Computer Science \& Engineering, \\ Sri Satya Sai University of Technology \& Medical Sciences, Sehore, Bhopal Indore Road, Madhya Pradesh, India \\ ${ }^{c}$ Research Co-Guide, Department of Information Technology, \\ Gokaraju Rangaraju Institute of Engineering \& Technology (Autonomous), Hyderabad
}

Article History: Received: 11 January 2021; Accepted: 27 February 2021; Published online: 5 April 2021

\begin{abstract}
Cloud computing is a promising worldview that gives computing resources as a service over a system. Various information replication approaches have been introduced for information cloud in the previous decades. All replication method access a few qualities, for example, adaptation to internal failure, versatility, dependability, execution, stockpiling utilization, information access time and so on. Late examination permitted us to more readily comprehend the cost drivers and significant choices that can influence the costs related with moving to the cloud, to make the RAND Cloud Cost Model, and to build up a structure for contrasting the cloud and other data stockpiling and the executives' options.
\end{abstract}

Introduction

Cloud computing is changing the IT business to flexibly admittance to their infrastructure and application services on a membership premise. Subsequently, numerous endeavors along with IBM, Microsoft, Google, and Amazon have started to give very surprising Cloud services to their clients. The layer of cloud service provider are empowered to get the necessities structure client. The client survey and solicitation are taken as contribution to give quality items. As of now, there is no predefined system for estimating client audit and offering great quality items. Cloud computing is related with nursing IT model that contains all vital computing parts and resources for creating and conveying cloud services. In the ongoing years, it has seen quick development, which might be influenced by numerous components. Most fundamentally, the cloud worldview encourages the strategy for gracefully and using computing resources and diminishes the costs. In a few nations with fitting net infrastructure, cloud-based services have apparently started to command diverse application regions. This is frequently generally noticeable inside the venture market. Also the, built up IT merchants are offering antiquated types of assistance (for example Microsoft work environment 365, Google mail), a few providers in square estimating services. These services must be finished and appropriately assessed before clients make a decision to utilize the items.

Cloud computing faces numerous difficulties and the difficulties are typify security, protection and trust. Among these, building trust between individuals from the cloud could be a significant issue that hampers inside and out utilization of cloud services. Basically, the customer data are gathered by the Cloud Service Provider's (CSP) data and clients have next to no administration over the techniques. Their data is gathered and processed in writing; be that as it may, inside the cloud computing setting it is as yet partner in nursing raising subject. In cloud computing environment every client esteems are impacted by trust based methodology and different publication results are gathered by Barber.

At the equipment level, the Cloud portrays the utilization of an assortment of dispersed services, applications, data and infrastructure included pools of PC, system, data and capacity resources. To qualify as a cloud environment, these parts ought to be provisioned to be quickly organized, gave, actualized and decommissioned utilizing an onrequest utility-like model of distribution and utilization.

At the point when a more defensive cloud computing arrangement must be offered, the principle standards to be engaged is the assurance of the kind of cloud to be figured it out. At present, four sorts of cloud sending models are accessible and they are the public cloud, the private cloud, the hybrid cloud and the network cloud. Every one of this cloud sending models can be clarified as demonstrated as follows.

Public cloud: It empowers the clients to reserve the privilege to utilize the cloud through interfaces, which include the standard internet browsers. This model ought to be paid for each utilization and it takes after the prepaid power metering framework that shows improved adaptability through the thought on spikes, which are fundamental for advancing the cloud. Subsequently, the customers in the cloud could meet their IT costs during the operational stage itself through the decrease in the chief cost spent on IT infrastructure.

Private cloud: This sort of cloud exists inside an in house undertaking datacenter of an association. It can uphold security, consistence and administrative essentials in a superior way and offers expanded degree of big business power over its abuse.

Hybrid cloud: This cloud has a place with the private cloud classification. Further, it has association with numerous outer cloud services such that it is controlled midway and expected as one unit, which is bound to a 
more defensive system. It makes a mix of the clouds that are public and private to yield virtual IT arrangements. With hybrid cloud, the information control is progressively defensive and the application can empower various gatherings to perform information access by means of the internet.

Community cloud: In this sort of cloud, the clouds work with a typical expectation. This cloud may help either a solitary or various associations and permits the data sharing, which is related to crucial, security, administrative consistence necessities and comparative different capacities, between the associations. Command over the community cloud is accomplished utilizing the constituent association (s) or through an outsider (Mell and Grance, 2011).

Regularly, cloud computing offers the infrastructure identified with both the equipment just as the software as services with the assistance of enormous server farms (Björkqvist et al., 2011). In like manner, the cloud computing has expanded the calculation just as the information stockpiling from the end client to endless server farm infrastructures. The Cloud infrastructure adjusts the equipment limit with respect to accomplishing the ideal nonfunctional Quality of Services (QoS). The cloud is monstrous and dynamic, with the goal that it is inconvenient to accomplish expanded dependability and effectiveness levels during the time the cloud server farms are gotten to. Moreover, the cloud computing framework permits various applications to be executed through the handling of gigantic measure of system information.

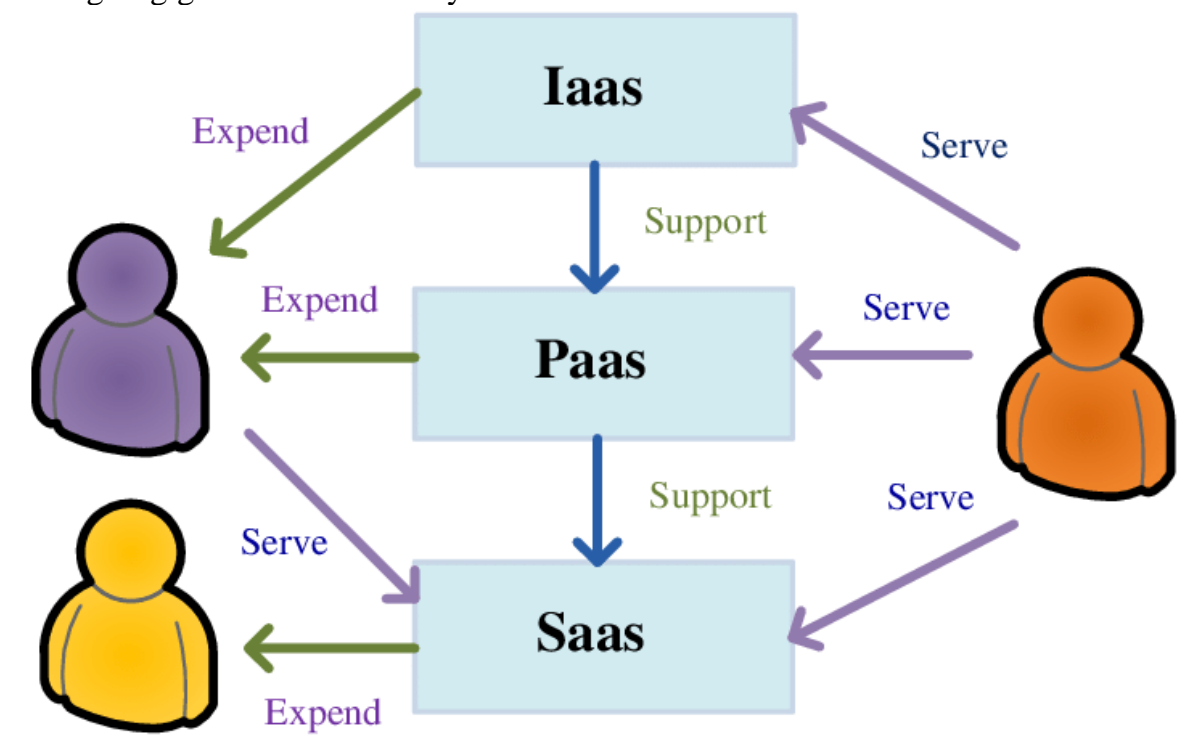

Figure 1. Cloud computing Structure

The network is made out of a few hubs. The equipment of the framework has more odds of harm because of this expanded number of hubs. At the point when the equipment gets harmed, the put away information in a hub will be additionally tainted. Further, an application mentioning the slammed information during its execution stage will neglect to have the information access from the ruined hub (Anjali and Rokade, 2014). Defilement keeps the applications from acquiring helpful and flourishing outcomes. Information replication is a novel strategy in cloud computing framework, which helps the applications in a ceaseless way through the avoidance of information debasement. Replication alludes to the strategy that is associated with offering different copies of a specific service at different hubs (Kemme and Alonso, 2010).

\section{Literature Review}

Cloud gives equipment normally a virtual server, as a service and make utilizing servers more helpful and cost proficient, as the meaning of cloud computing given by NIST is "a compensation examine model for empowering accessible, advantageous and on request network admittance to a mutual pool of configurable computing resources (e.g., networks, servers, stockpiling, applications, services) that can be quickly provisioned and delivered with insignificant administration exertion or service provider association." Here we reviewed a few papers identified with cost effectiveness in cloud.

Fei Tao et al. proposed the issue of dynamic immigration of Virtual Machines. The proposed DM-VM thinks about imperativeness consumption, VMs correspondence, and takes immigration charges under the conditions. 
David Candeia et al. announced that utilizing reservation market's cases, the cloud providers can diminish their usable cost, where the occasions can be bought. However, they additionally need to figure the amount of occasions required in the long haul. Their work explores the significance of scope quantification. Also, the effect of business driven heuristics is introduced.

Xuanjia Qiuet et al. examine that in hybrid cloud, the content distribution service can be an ideally relocated. The creator has proposed a system, which depends on Lyapunov enhancement hypothesis. To lessen the employable cost and ensured QoS, a consolidated algorithm for dynamic, joint content situation and solicitation distribution is proposed.

Shuangcheng Niu et al. proposed a computing model for associations named as Semi-Elastic Cluster (SEC) for holding and progressively resizing a virtual bunch in cloud. The creator has introduced cluster scheduling set and an algorithm for saved case provisioning, which uses work history is likewise introduced.

Philipp Hoenisch et al. introduced a novel path for business measures scheduling and enhances the operational cost of resources. For that, they bring up the issue of productive scheduling of service cases among resources.

Yi Zhang et al. proposed a heuristic technique that contains requesting and task arranging of assignment. It uses Longest Task First (LTF) plan to create an assignment request. A technique for Task Assignment is fixed for planning undertakings in required request each in turn. The trial result shows that the proposed EH strategy has demonstrated preferable outcomes over the pattern (Round Robin) impressively.

Renan Delvalle et al. suggested that the server farms go through an extensive cost for force and vitality utilization, while running these workloads and overseeing high usage of their segments. To manage this cost they have imagined another structure, called Electron. The proposed new structure is expected to elite, proficient force and scheduling procedures.

Eya Dhib et al. proposed a structure to manage resource provisioning in cloud .Their proposed structure is planned to deal with the tradeoff between cost associated with resource distribution and preparing delay and furthermore allots important virtual resources.

Mohammad Jassas et al. introduced a structure for installed frameworks, to build the use of limited nearby. The structure gives adaptability and throughput. The scheduling algorithm lessens the execution time and assessed cost.

Gursleen Kaur et al. proposed a hybrid hereditary algorithm to lessen the operational cost and execution time under the given cutoff time .

Xuezhi Zeng et al. proposed a scheduling algorithm (MASA) which depends on voracious methodology. The methodology praises the client's to reduce the leasing cost of resources and thinking about client's financial plan and cutoff time imperatives. The outcome shows significant decreased cost as contrasted and other current techniques.

Lei Jiao et al. inspected the issue of value improvement in the event of dynamic OSN over consecutive timespan. They structure the cost, the QoS, and the information accessibility of OSN.

Yichao Jin et al. examined the cost ideal content position issue. Especially, it is intended to diminish the whole cost, concerning the resource limit.

Melody Li et al. zeroed in on improving the utilitarian cost of hybrid cloud model by likely assessing the trouble. Their method can manage the issues where leasing costs and client's up and coming likelihood distribution are obscure.

Yongyi Ran et al. made a strategy to diminish the whole computing cost considering QoS by utilizing number of the bought occurrences powerfully. By planning QoS, the creator has proposed a plan for dynamic example provisioning to figure the base cases for the up and coming requests.

Peng Zhao et al. explored to lessen the drawn out vitality charge of dynamic Internet-scale frameworks. They planned a powerful methodology of solicitation planning to determine issue, while balancing the tradeoff in vitality cost and defer execution. In light of present line accumulations and framework expresses, the algorithm takes ongoing choices.

Jianguo Yao et al. proposed a structure COMIC, for example Cost Optimization for Internet Content Multihoming. The creator has structured a unified enhancement approach for content Multihoming environment, to adjust end client's loads powerfully and to decrease the content service charges.

Soumen Moulik et al. announced that they endeavor to take out such business from the valuing arrangements, and anticipate this challenge .To accomplish it they use bartering idea engaged with game hypothesis .

Yusen Li et al. announced that new form of DBP issue, named as MinTotal DBP issue whose goal is to limit all out receptacle cost .It is dictated by the issue of solicitation sending created from gaming frameworks in cloud . Wenhua Xiao et al. revealed advancement issue and planned an online algorithm. Keeping up the client's QoE their technique can diminish the cost to lease resources on cloud.

Abhishek Gupta et al. recommended the methodology for choice of cloud for HPC and the HPC applications that sudden spike in demand for cloud. They showed by running a gathering of HPC applications and broke down their cost and execution. 
Danilo Ardagna et al. proposed a game technique for dealing with the resources progressively. The strategy shows a cost model indicating their income just as punishments because of disappointments of solicitations.

Tamir Hegazy et al. revealed that the cost and time can be spared generally because of the virtualization of regulators and the minimization of equipment charge.

Boyang Yu et al. detailed the secluded memory store's issues related with demand dispatching. To keep up line strength, least vitality cost and high reserve hit, an online technique is proposed.

Balaji Palanisamy et al. introduced Cura, to give cost effective MapReduce services in cloud. Cura is more proficient when contrasted with existing cloud services.

Hamed Shah Mansouri et al. proposed task scheduler to accomplish the evaluating plan for cell phones. Furthermore, they planned an answer for consider vitality cost, service cost, deferral and scheduling.

Shivaswamy Rashmi and Anirban Basu proposed a scheduler to streamline the cost of VM, abandwidth and vitality, and for work execution under the cutoff time.

Arvind Mohan et al. proposed an elite BARENT work process scheduler. The scheduler expects to decrease the work process make length and works in various cloud environment.

Qiushi Wang et al. built up a quick and productive system to compute occurrences for each estimating choice on hourly distribution premise. The creator additionally structured a plan for appropriate course of action of booked occurrences.Profit Maximization at Cloud

K.Sutha et al. examined a profit augmentation plan to comprehend significance of occupation scheduling for effective usage of services.

Qiufen Xia et al. mean to enhance the operational cost, with limit of resources and client reasonableness imperatives to improve framework throughput.

Subarna Chatterjee et al. introduced a model, which manages profit expansion and dynamic valuing methodology for equipment

Xiaoxi Zhang et al. structured a capable online VM sell off based plan with differing spans and area inclinations, and meaning to government assistance and profit amplification of cloud.

Laiping Zhao et al. introduced an online VM situation algorithm to amplify the income of cloud provider by dropping SLA infringement cost.

Jianxiong Wan et al. considered an issue of estimating of dynamic server dependent on resource needs.

Mahyar Movahed Nejad et al. detailed covetous and ideal answer for provisioning of VM powerfully and apportioning the resources in a bartering based model to winning client and as needs be figures their installments.

\section{Conclusion}

Cloud computing has become to be an inexorably conspicuous region for innovative work. From cloud master viewpoint, it is important to enhance the cost and augment the profit, while from client perspective the exhibition and throughput ought to be high, remembering the least valuing technique offered by the cloud provider. Overseeing cost and better portion of resources can be accomplished by top to bottom investigation of cloud and its workload. We summed up this literature by deliberate development of resource scheduling with cost improvement and profit expansion parameter.

\section{References}

1. S. K. Sonkar, "A Review on Resource Allocation and VM Scheduling Techniques and a Model for Efficient Resource Management in Cloud Computing Environment”, IEEE, pp. 1-7, 2016.

2. Jing Mei, Kenli Li, Aijia Ouyang and Keqin Li, "A Profit Maximization Scheme With Guaranteed Quality Of Service In Cloud Computing”, IEEE Transactions On Computers Vol: Pp No: 99 , Pp. 1-14, 2015

3. Kumar, A., Vengatesan, K., Rajesh, M., \& Singhal, A. (2019). Teaching literacy through animation \& multimediaInt. J. Innovative Technol. Exploring Eng, 8(5), 73-76

4. Fei Tao, Chen Li, T. Warren Liao, And Yuanjun Laili, "BGMBLA: A New Algorithm For Dynamic Migration Of Virtual Machines In Cloud Computing”, IEEE Transactions On Services Computing, Vol. 9, No. 6, , Pp.1-16,2016.

5. David Candeia, Ricardo Araujo Santos and Raquel Lopes, "Business-Driven Long-Term Capacity Planning for Saas Applications”, IEEE Transactions on Cloud Computing, Vol. 3, No. 3, Pp1-14, 2015.

6. Xuanjia Qiu, Hongxing Li, Chuan Wu, Zongpeng Li and Francis C.M. Lau, "Cost-Minimizing Dynamic Migration Of Content Distribution Services Into Hybrid Clouds", IEEE Transactions on Parallel And Distributed Systems, Vol. 26, No. 12, Pp.1-16,2015.

7. Shuang cheng Niu, Jidong Zhai, Xiaosong Ma, Xiongchao Tang, Wenguang Chen, and Weimin Zheng, "Building Semi-Elastic Virtual Clusters For Cost-Effective Hpc Cloud Resource Provisioning" IEEE Transactions On Parallel And Distributed Systems, Vol. 27, No. 7, Pp.1-14,2016. 
8. Philipp Hoenisch, Dieter Schuller, Stefan Schulte, Christoph Hochreiner, and Schahram Dustdar, "Optimization Of Complex Elastic Processes", IEEE Transactions On Services Computing, Vol. 9, No. 5,Pp. 1-14,2016.

9. Renan Delvalle, "Electron: Towards Efficient Resource Management on Heterogeneous Clusters with Apache Mesos", IEEE 10th International Conference on Cloud Computing, pp.1-8, 2017.

10.Eya Dhib, "Resources allocation trade-off between cost and delay over a distributed Cloud infrastructure", 7th International Conference on Sciences of Electronics, Technologies of Information and Telecommunications (SETIT), pp.1-5, 2016.

11.Mohammad Jassas, "A Framework for Extending Resources of Embedded Systems using the Cloud", IEEE 30th Canadian Conference on Electrical and Computer Engineering pp.1-5, 2017.

12.Gursleen Kaur," Deadline Constrained Scheduling of Scientific Workflows on Cloud using Hybrid Genetic Algorithm”, IEEE, pp.1-5, 2017.

13.Ryan Marcus, "A Learning-based Service for Cost and Performance Management of Cloud Databases", IEEE 33rd International Conference on Data Engineering, pp.1-2, 2017.

14.Sayyad, S., Mohammed, A., Shaga, V., Kumar, A., \& Vengatesan, K. (2018). Digital Marketing Framework Strategies Through Big Data. In International conference on Computer Networks, Big data and IoT (pp. 1065-1073)

15.Xuezhi Zeng, "SLA-aware Scheduling of Map-Reduce Applications on Public Clouds", IEEE 18th International Conference on High Performance Computing and Communications; IEEE 14th International Conference on Smart City; IEEE 2nd International Conference on Data Science and Systems, pp.1-8,2016

16.Lei Jiao, Jun Li, Tianyin Xu, Wei Du and Xiaoming Fu, "Optimizing Cost For Online Social Networks On Geo-Distributed Clouds", IEEE/ACM Transactions on Networking, Vol. 24, No. 1, February, Pp. 114, 2016.

17.Yichao Jin, Yonggang Wen and Kyle Guan, "Toward CostEfficient Content Placement in Media Cloud: Modeling and Analysis", IEEE Transactions on Multimedia, Vol. 18, No. 5, Pp.1-13, 2016.

18.Song Li, Yangfan Zhou, Lei Jiao, Xinya Yan, Xin Wang and Michael Rung-Tsong Lyu, "Towards Operational Cost Minimization in Hybrid Clouds for Dynamic Resource Provisioning with DelayAware Optimization”, IEEE Transactions On Services Computing, Vol. 8, No. 3, Pp.1-12, 2015. 